\title{
Mean Energy Density of Photogenerated Magnetic Fields Throughout the EoR
}

\author{
Jean-Baptiste Durrive ${ }^{1,2}$, Hiroyuki Tashiro' ${ }^{2}$, Mathieu Langer ${ }^{1}$ \\ and Naoshi Sugiyama ${ }^{2,3,4}$ \\ ${ }^{1}$ Institut d'Astrophysique Spatiale, CNRS, UMR 8617, Univ. Paris-Sud, Université \\ Paris-Saclay, IAS, Bât. 121, 91405, Orsay, France \\ email: jean.baptiste.durrive@e.mbox.nagoya-u.ac.jp \\ ${ }^{2}$ Department of Physics and Astrophysics, Nagoya University, Nagoya 464-8602, Japan \\ ${ }^{3}$ Kobayashi-Maskawa Institute for the Origin of Particles and the Universe, \\ Nagoya University, Nagoya 464-8602, Japan \\ ${ }^{4}$ Kavli Institute for the Physics and Mathematics of the Universe (Kavli IPMU), \\ The University of Tokyo, Chiba 277-8582, Japan
}

\begin{abstract}
There seems to be magnetic fields at all scales and epochs in our Universe, but their origin at large scales remains an important open question of cosmology. In this work we focus on the generation of magnetic fields in the intergalactic medium due to the photoionizations by the first galaxies, all along the Epoch of Reionization. Based on previous studies which considered only isolated sources, we develop an analytical model to estimate the mean magnetic energy density accumulated in the Universe by this process. In our model, without considering any amplification process, the Universe is globally magnetized by this mechanism to the order of, at least, several $10^{-18} \mathrm{G}$ during the Epoch of Reionization (i.e. a few $10^{-20} \mathrm{G}$ comoving).
\end{abstract}

Keywords. magnetic fields, methods: analytical, cosmology: theory

\section{Introduction}

The question of the origin of cosmological magnetic fields (CMF) remains an important open question of cosmology. Many magnetogenesis mechanisms have been proposed (cf. for example Ryu et al. (2012) or Widrow et al. (2012) for reviews). It is usual to classify them chronologically, with on the one hand those operating in the primordial universet and on the other hand the post-Recombination onesł. However, none of them is preferred so far.

The evolution of CMF through structure formation (i.e. essentially their amplification from generation to once we observe them) is not settled yet, but the current status is that a priori a seed field of strength between $10^{-22} \mathrm{G}$ and $10^{-12} \mathrm{G}$ would be enough to account for the observed fields in structures, after amplification processes. Now, due to turbulence, the magnetic fields inside structures lost their initial properties. On the contrary, magnetic field seeds in the intergalactic medium (IGM) probably evolved less drastically. Therefore, it is paramount to understand the possible generation of intergalactic magnetic fields, as they may be our key to probing the generation of CMF.

This has precisely been the motivation of Durrive \& Langer (2015). They studied a mechanism which generates CMFs in the neutral IGM by the first luminous sources

$\dagger$ During inflation, at the electroweak and quark-hadron phase transitions, or during Recombination

$\ddagger$ Thermal Biermann battery, plasma instabilities or radiation-based processes, but fields may also in fact originate from outflows 

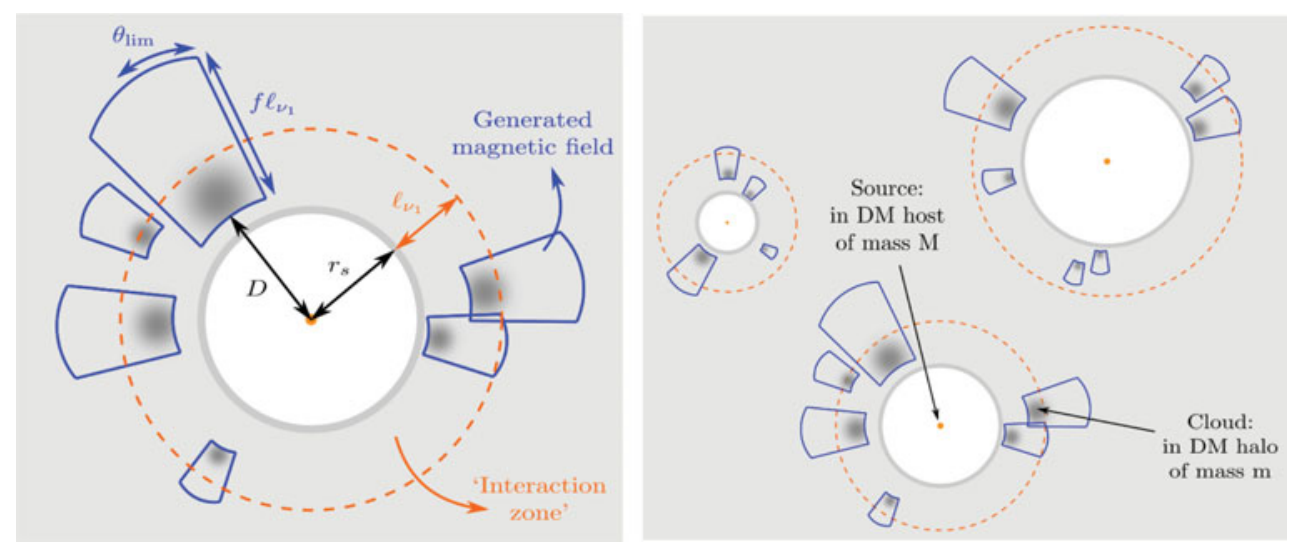

Figure 1. Illustration of our procedure: We first evaluate the magnetic field generated around one source, surrounded by a distribution of clouds (left panel) and then we consider a distribution of sources (right panel). See section 2 for the meaning of the colors and lines.

(Population III stars, primordial galaxies and quasars) that form all along EoR. Physically the process is natural: since the mean free path of photons goes as the cube of their energy, high energy photons (UV and X) reach large distances, well beyond the Stromgren spheres of the source they are from. As they ionize the neutral IGM they generate a charge separation, thus inducing an electric field, which is maintained since the source emits photons continuously. In regions where this electric field is rotational, by Maxwell-Faraday's law, we know that some magnetic field is induced. As detailed in their paper, this latter condition is fulfilled in regions with density gradients, i.e. magnetic fields appear in the IGM because it is full of inhomogeneities.

The purpose of Durrive \& Langer (2015) was to derive the details of this mechanism around isolated sources. In our work here we consider in addition the cosmological context, and estimate to which level this mechanism may have participated to the magnetization of the whole IGM throughout the EoR.

\section{Procedure}

We illustrate our procedure in Fig 1, which consists in the following steps:

(a) First, we consider an isolated source, cf. the orange dot in the left panel of Fig 1. In this figure, the gray blurry spots represent the small inhomogeneities of the IGM, namely small overdense regions of baryonic gas that surround the source. In Durrive \& Langer (2015) the authors derived in great details the characteristics of the magnetized region around each of such baryonic clouds. We represent this as blue frames in Fig 1. For our purposes, we do not need so much details, so we first condensed these information into a simpler and more tractable version of their results. Thanks to this, we then derive an efficient expression for the magnetic energy density $E_{m}(D)$ associated to any cloud of mass $m$ at a given distance $D$ to the source.

(b) We then sum up the contribution of all the clouds surrounding the source. But to do so, we need to estimate the distribution of such baryonic clouds, which we do by considering their underlying dark matter (DM) haloes, in order to make use of the Press-Schechter formalism. In other words: The source, contained inside a DM halo of 
mass $M$, contributes to the magnetization of the IGM by injecting an energy

$$
E_{M}=\int_{r_{s}}^{r_{s}+\ell_{\nu_{1}}} \int_{m_{\min }}^{m_{\max }} E_{m}(D) \mathrm{d}^{2} P(D, m \mid M)
$$

where $\mathrm{d}^{2} P(D, m \mid M)$ is the probability of finding a DM cloud of mass $m$ within a spherical shell of volume $4 \pi D^{2} \mathrm{~d} D$, at a distance $D$ from the halo of mass $M$. As one can see from the boundaries of the first integral in equation (2.1), we take into account only the clouds outside the Stromgren sphere (of radius $r_{s}$ ), but not too far from it, namely inside an 'interaction zone' beyond which not enough photoionizations occur for the mechanism to be efficient (orange dashed lines in Fig 1). The boundaries of the second integral, $m_{\min }$ and $m_{\max }$ for the mass of the clouds, are important parameters of this process, and we discuss in details how we chose them in Durrive et al. (2017).

(c) Then we consider the full cosmological context, as in the right panel of Fig 1. The energy $E_{M}$ generated around each source must be integrated over the distribution of DM haloes containing these sources. However, ionized bubbles of individual sources start to overlap around the end of the EoR. Since our mechanism takes place only in the neutral IGM, the efficiency of magnetic field generation actually decreases as reionization proceeds. Hence, let us now denote by $Q_{i}(z)$ the volume filling factor $\dagger$ of ionized bubbles at a given redshift $z$. While summing up the contribution of each single source, we weigh this with a factor $1-Q_{i}(z)$ in order to reduce the generated magnetic field energy as time increases according to the ionization of the IGM. We thus estimate the mean physical magnetic energy density generated by photoionizations during EoR as

$$
\frac{B_{\mathrm{p}}^{2}(z)}{8 \pi}=(1+z)^{4} \int_{z}^{z_{0}} \mathrm{~d} z^{\prime} \frac{1-Q_{i}}{\left(1+z^{\prime}\right)^{5} H} \int_{M_{*}}^{M_{\max }} \mathrm{d} M E_{M} g_{\mathrm{gl}} \frac{\mathrm{d} n_{M}}{\mathrm{~d} M}
$$

where $\frac{\mathrm{d} n_{M}}{\mathrm{~d} M}$ is the mass function of the DM haloes, with mass $M$, hosting the sources. We also use the Press-Schechter formalism to compute it and we discuss our choices for the values of the boundaries $M_{*}$ and $M_{\max }$. The parameter $z_{0}$ is the redshift at which the first sources form. We also introduce a parameter $g_{\mathrm{gl}}$ corresponding to the rate at which DM haloes 'switch on' sources, with a numerical value chosen in order for our model to be consistent with important observational constraints on EoR, namely the optical depth parameter deduced from the Planck 2015 data and the end of EoR. Finally, the parameter $H$ and the $(1+z)$ factors are due to the expansion of the Universe, which dilutes the magnetic fields.

\section{Results and discussion}

Formula (2.2) constitutes our main result, together with Fig 2 which shows examples of the redshift evolution of the mean comoving magnetic field thus accumulated in the IGM. The general trends are natural: As time passes, this value increases since the sources appearing magnetize the IGM, and the curves reach a plateau at redshift $\sim 8$ as EoR ends and no more neutral IGM is available for this mechanism to operate. The different curves correspond to three types of models. The green curve corresponds to our fiducial model, the red is for a universe with 'strongly ionizing' galaxies (i.e. maximal escape fraction and stars formed to stay consistent with Planck data) while the blue is for a universe with 'weakly ionizing' galaxies (i.e. minimal escape fraction and stars formed).

We conclude that the Universe may thus be globally magnetized to the order of a few $10^{-20} \mathrm{G}$ comoving i.e. several $10^{-18} \mathrm{G}$ during EoR. We emphasize that when comparing

$\dagger$ which we estimate in the usual way done in studies of EoR 


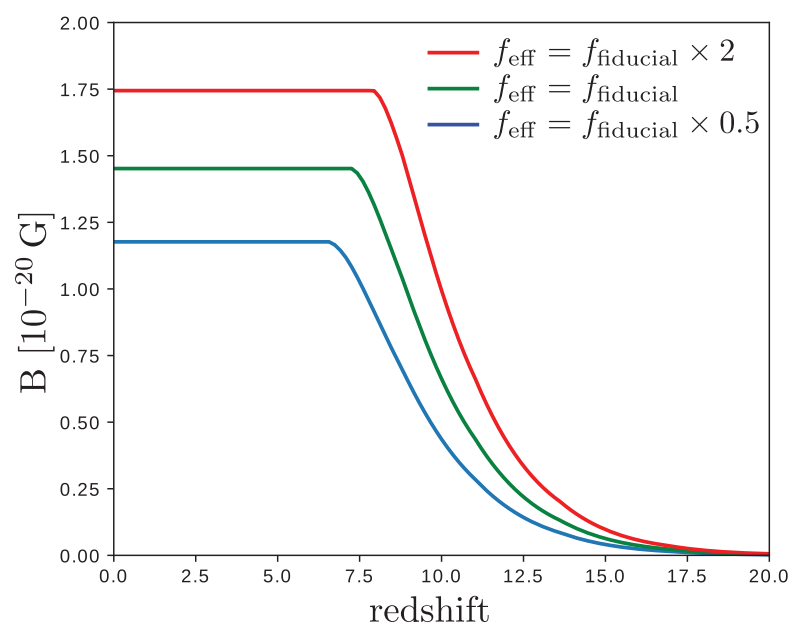

Figure 2. Evolution with redshift of the mean comoving magnetic field accumulated in the IGM for different reionization parameters. See section 3 for details on the colors.

our predicted values to other studies in the literature, one should remember that we did not take into account any amplification process. See Durrive et al. (2017) for more details on comparisons, but the bottom line is that we claim that while the magnetogenesis process itself is of astrophysical nature, it is relevant on cosmological scales, and in particular in voids.

Of course, this analysis is simplistic in several ways, but we believe that it constitutes a good order of magnitude because our approach overestimates some processes and underestimates others [cf. discussions in Durrive et al. (2017)]. The point of such an analytic approach is also to exhibit the numerous processes at play, which is key to guide us in the next step, namely of using numerical simulations, to assess the properties of the fields in more realistic contexts (work in progress). Let us end with optimism, by pointing out that some recent studies suggest that even fields as weak and as remote as the ones predicted here, maybe one day, will in fact be directly measurable with SKA [cf. Venumadhav et al. (2017) and Gluscevic et al. (2017)].

Acknowlegments JBD acknowledges financial support by the P2IO LabEx (ANR-10LABX-0038) in the framework 'Investissements d'Avenir' (ANR-11-IDEX-0003-01) managed by the French National Research Agency (ANR). This work was supported in part by JSPS Grants-in-Aid for Scientific Research under Grant Nos. 25287057 (N.S.), 15H05890 (N.S.) and 15K17646 (H.T.).

\section{References}

Durrive, J.-B. \& Langer, M., 2015, 1993, MNRAS, 453, 345

Durrive, J.-B., Tashiro, H., Langer, M. \& Sugiyama, N. 2017, MNRAS, 472, 1649

Gluscevic, V., Venumadhav, T., Fang, X., Hirata, C., Oklopčcić, A. \& Mishra, A. 2017, Phys. Rev. D, 95, 083011

Ryu, D., Schleicher, D. R. G., Treumann, R. A., Tsagas, C. G. \& Widrow, L. M. 2012, Space Sci. Revs, 166, 1

Venumadhav, T., Oklopčcić, A., Gluscevic, V., Mishra, A. \& Hirata, C. M. 2017, Phys. Rev. D, 95, 083010

Widrow, L. M., Ryu, D., Schleicher, D. R. G., Subramanian, K., Tsagas, C. G. \& Treumann, R. a. 2012, Space Sci. Revs, 166, 37 